\title{
Expression and secretion of CXCL12 are enhanced in autosomal dominant polycystic kidney disease
}

\author{
Hyunho Kim ${ }^{1}$, Jinmo Sung ${ }^{1}$, Hyunsuk Kim ${ }^{2}$, Hyunjin Ryu ${ }^{3}$, Hayne Cho Park ${ }^{4}$, Yun Kyu Oh ${ }^{5}$, Hyun-Seob Lee ${ }^{6}$, \\ Kook-Hwan $\mathrm{Oh}^{3}$ \& Curie Ahn, \\ ${ }^{1}$ Center for Medical Innovation, Biomedical Research Institute, Seoul National University Hospital, Seoul 03082, ${ }^{2}$ Internal Medicine, \\ Hallym University Medical Center, Chuncheon Sacred Heart Hospital, Chuncheon 24253, ${ }^{3}$ Department of Internal Medicine, Seoul \\ National University College of Medicine, Seoul 03080, ${ }^{4}$ Department of Internal Medicine, Hallym University Medical Center, Kangnam \\ Sacred Heart Hospital, Seoul 07441, ${ }^{5}$ Department of Internal Medicine, Seoul National University Boramae Medical Center, Seoul 07061, \\ ${ }^{6}$ Genomics Core Facility, Department of Transdisciplinary Research and Collaboration, Biomedical Research Institute, Seoul National \\ University Hospital, Seoul 03082, Korea
}

\begin{abstract}
Autosomal dominant polycystic kidney disease (ADPKD), one of the most common human monogenic diseases (frequency of 1/1000-1/400), is characterized by numerous fluid-filled renal cysts (RCs). Inactivation of the PKD1 or PKD2 gene by germline and somatic mutations is necessary for cyst formation in ADPKD. To mechanistically understand cyst formation and growth, we isolated RCs from Korean patients with ADPKD and immortalized them with human telomerase reverse transcriptase (hTERT). Three hTERT-immortalized RC cell lines were characterized as proximal epithelial cells with germline and somatic PKD1 mutations. Thus, we first established hTERT-immortalized proximal cyst cells with somatic PKD1 mutations. Through transcriptome sequencing and Gene Ontology (GO) analysis, we found that upregulated genes were related to cell division and that downregulated genes were related to cell differentiation. We wondered whether the upregulated gene for the chemokine CXCL12 is related to the mTOR signaling pathway in cyst growth in ADPKD. CXCL12 mRNA expression and secretion were increased in RC cell lines. We then examined CXCL12 levels in RC fluids from patients with ADPKD and found increased CXCL12 levels. The CXCL12 receptor CXC chemokine receptor 4 (CXCR4) was upregulated, and the mTOR signaling pathway, which is downstream of the CXCL12/CXCR4 axis, was activated in ADPKD kidney tissue. To confirm activation of the mTOR signaling pathway by CXCL12 via CXCR4, we treated the RC cell lines with recombinant CXCL12 and the CXCR4 antagonist AMD3100; CXCL12 induced the mTOR signaling
\end{abstract}

*Corresponding author. Tel: +82-2-2072-2222; Fax: +82-2-741-4876; E-mail: curie@snu.ac.kr

https://doi.org/10.5483/BMBRep.2019.52.7.112

Received 17 April 2019, Revised 6 May 2019, Accepted 23 May 2019

Keywords: Autosomal dominant polycystic kidney disease (ADPKD), CXCL12, CXCR4, Cyst, hTERT pathway, but the CXCR4 antagonist AMD3100 blocked the mTOR signaling pathway. Taken together, these results suggest that enhanced CXCL12 in RC fluids activates the mTOR signaling pathway via CXCR4 in ADPKD cyst growth. [BMB Reports 2019; 52(7): 463-468]

\section{INTRODUCTION}

Progressive renal cyst (RC) growth in autosomal dominant polycystic kidney disease (ADPKD), one of the most common genetic diseases with a frequency of 1 in 400 to 1000 individuals, is associated with loss of polarity, abnormal proliferation and increased fluid secretion, ultimately leading to renal failure $(1,2)$. ADPKD is caused by mutations in the PKD1 or PKD2 genes and their protein products polycystin-1 and polycystin-2, respectively, which function as calcium channels via the polycystin-1/polycystin-2 complex and regulate multiple signaling pathways, including the JAK/STAT, mTOR, extracellular signal-related kinase (ERK), and Wnt signaling pathways, to maintain normal tubular structure $(3,4)$.

Unfortunately, there are no mutation hot spots in either gene. Numerous germline mutations in PKD1 and PKD2 have been identified $(5,6)$. In addition to a germline mutation, in a second hit model, a somatic mutation occurs in the remaining normal allele, leading to cyst formation. PKD1 or PKD2 inactivation caused by somatic mutation induces abnormal renal epithelial cell proliferation and the loss of polarity, resulting in cyst formation. In this second hit model of cyst formation in ADPKD, the timing and type of somatic mutations in the PKD genes affect disease severity and the onset of end-stage renal disease (ESRD) (7).

Telomere shortening that occurs in normal dividing cells is related to the aging and mortality of primary cells. Exogenous human telomerase reverse transcriptase (hTERT) maintains telomeres, making primary cells immortal $(8,9)$. In previous reports, primary human cells, including fibroblasts, endothelial 
cells and mammalian epithelial cells, were immortalized by hTERT alone (10). Immortalization using hTERT ensures minimal cancer-associated changes and altered phenotypic properties $(11,12)$.

CXCL12, also known as stromal cell-derived factor 1 (SDF-1), is an inducible chemokine and one of the most important CXC chemokines. CXCL12 comprises 68 amino acids with 9 exons and is expressed in multiple normal tissues and serum. The CXCL12 promoter has three GC boxes and one CAAT box that are binding sites for the transcription factors SP1 and CTF, respectively (13). CXCL12 plays a role in biological effects through binding to its receptors CXC chemokine receptor 4 (CXCR4) and CXC chemokine receptor 7 (CXCR7) $(14,15)$. CXCR4 comprises 352 amino acids with two exons and is expressed in multiple cell types, including stromal fibroblasts, lymphocytes, cancer cells, epithelial cells and endothelial cells. The CXCL12/CXCR4 interaction constitutes a biological axis that induces the angiogenesis, growth and metastasis of cancers (16). The CXCL12/CXCR4 axis plays an important role in proliferation, cell survival, migration and chemotaxis by activating several downstream signaling pathways, such as the mTOR, ERK1/2 and p38 pathways (17-19).

In this study, we demonstrated the immortalization of ADPKD RC-derived cells with somatic mutations by hTERT, and CXCL12 upregulation was identified by transcriptome sequencing analysis. Furthermore, we suggest that the CXCL12/CXCR4 axis might regulate the mTOR signaling pathway in ADPKD cyst growth.

\section{RESULTS}

hTERT-immortalized ADPKD renal cyst ( $R C$ )-derived cells were characterized as proximal tubular epithelial cells RC-derived cells are useful in studying RC formation and growth in ADPKD, but primary cells have limited proliferative capacity in cell culture. Therefore, we immortalized ADPKD RC cells. We isolated cysts from the kidneys of three Korean ADPKD patients, and primary cultured cyst-derived cells were immortalized by hTERT. We selected hTERT as an immortalization agent because immortalization by SV40 T antigen alters several proliferative signaling pathways and induces genomic instability, but immortalization by hTERT has relatively little impact on these signaling pathways and genomic stability.

After ADPKD RC cell immortalization, we investigated whether the immortalized cell lines were derived from cystic epithelial cells or fibroblasts. These established ADPKD cyst cell lines expressed cytokeratin, which is an epithelial marker (Fig. 1A). To identify the tubule origins of the ADPKD RC cell lines, we used the nephron segment-specific markers FITC-conjugated anti-lotus tetragonolobus lectin (LTL) specific for proximal tubules and rhodamine-conjugated anti-dolichos biflorus agglutinin (DBA) specific for collecting ducts. All of our ADPKD renal cell lines were LTL-positive and DBA- negative, indicating that they originated from proximal tubules (Fig. 1B). Thus, hTERT-immortalized ADPKD RC cell lines were characterized as proximal tubular epithelial cells.

\section{Genetic analysis revealed somatic mutations in the PKD1} gene in ADPKD RC cell lines

Direct sequencing of genomic DNA (gDNA) from the blood paired with gDNA from the ADPKD RC cell lines from the same patients was conducted to identify both germline and somatic mutations in the ADPKD RC cell lines. We classified mutations found in both the blood and RC cell lines as germline mutations and mutations found only in the RC cell line as somatic mutations (Table 1).

Based on the ADPKD database (PKDB; http://pkdb.mayo.edu),

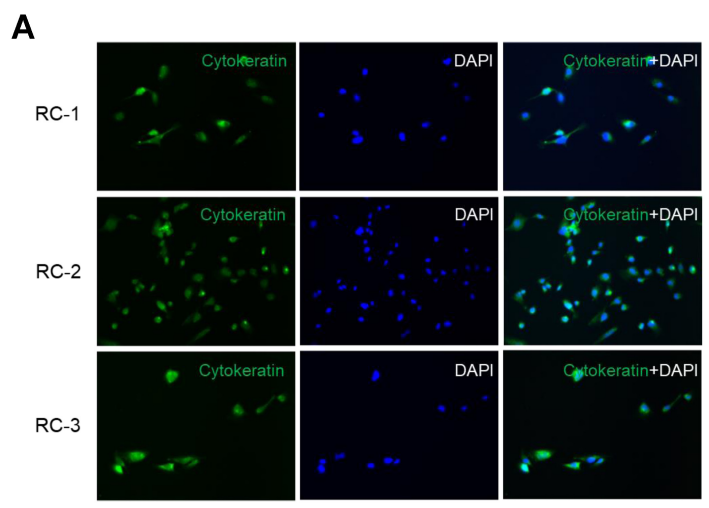

B

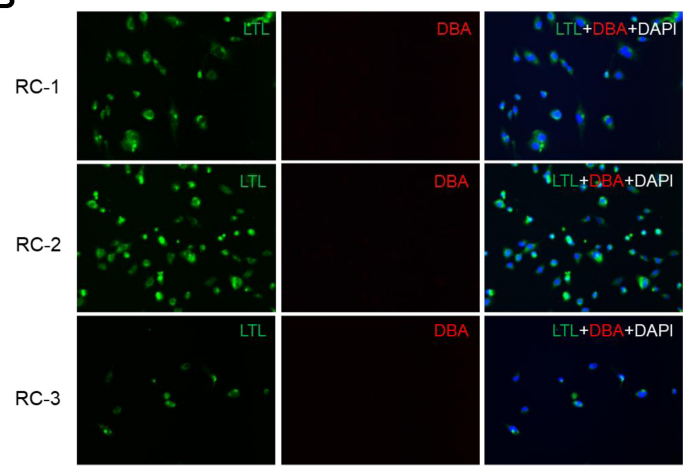

Fig. 1. Immortalized autosomal dominant polycystic kidney disease (ADPKD) renal cyst (RC) cell lines were derived from proximal tubular epithelial cells. (A) The epithelial origins of the RC cell lines were determined by cytokeratin staining. The three RC cell lines from different ADPKD patients were incubated with FITC-labeled pancytokeratin (green), followed by counterstaining with DAPI (blue). (B) The tubule origins of the RC cell lines were analyzed with nephron segment-specific markers. The RC cell lines were incubated with FITC-conjugated anti-lotus tetragonolobus lectin (LTL, green) and rhodamine-conjugated anti-dolichos biflorus agglutinin (DBA, red). Nuclei were counterstained with DAPI (blue). All RC cell lines showed LTL-positive and DBA-negative staining, indicating proximal tubule origins. 
Table 1. Genetic analysis of $P K D 1$ gene in cyst-derived immortalized cell lines

\begin{tabular}{|c|c|c|}
\hline $\begin{array}{l}\text { ADPKD Cyst cell } \\
\text { line }\end{array}$ & Germline mutation & Somatic mutation \\
\hline RC-1 & $\begin{array}{c}\text { c. } 11614 G>T \\
(p . E 3872 X)\end{array}$ & $\begin{array}{l}\text { c. } 4412 \text { 4415delTCAA } \\
\text { (p.I1471RfsX62) }\end{array}$ \\
\hline RC-2 & $\begin{array}{l}\text { c. } 9924-3 C>G \\
\text { (IVS29-3C > G) }\end{array}$ & $\begin{array}{c}\text { c.3504_3507delTGTC } \\
\text { (p.V1169fsX) }\end{array}$ \\
\hline RC-3 & $\begin{array}{l}\text { c.8347G >C } \\
\text { (p.A2783P) }\end{array}$ & $\begin{array}{l}\text { c.6891C>G } \\
\text { (p.H2297Q) }\end{array}$ \\
\hline
\end{tabular}

RC: Renal Cyst cells.

the RC-1 (E3872X) and RC-2 (IVS29-3C > G) germline mutations are known to be pathogenic, but the RC-3 (A2783P) germline mutation is novel. To elucidate whether the RC-3 germline mutation is pathogenic, we used PolyPhen-2 (http://genetics. bwh.harvard.edu/pph2), a tool that predicts the possible impact of amino acid substitution on the function of human proteins. By this prediction algorithm, the RC-3 germline mutation was predicted to most likely be damaging, with a score of 1.000 . This prediction was further confirmed with Mutation Taster (http://www.mutationtaster.org) and SIFT (https://sift.bii.a-star.edu.sg/). Thus, the germline mutations in ADPKD RC cell lines were characterized as nonsense mutations, intervening sequence (IVS) silent mutations, or missense mutations of the PKD1 gene. Somatic mutations in the PKD1 gene were found in only the ADPKD RC cell lines and different from PKD1 gene germline mutations, indicating a second hit model of cyst formation. Taken together, these results suggest that our immortalized cell lines were derived from ADPKD RCs without contamination from cells neighboring the RC that had only a germline mutation.

\section{mRNA expression and secretion of the chemokine CXCL12 were increased in ADPKD RC cell lines}

To investigate differential gene expression in ADPKD RCs, we performed transcriptome sequencing of ADPKD RC cell lines, which showed many differentially expressed genes between normal proximal tubule cells and ADPKD RC cell lines (Fig. 2A). After normalization, 751 genes were significantly upregulated, and 558 genes were significantly downregulated in all three RC cell lines. As shown by Gene Ontology (GO) analysis of the biological process (BP) terms of the upregulated and downregulated genes, the upregulated genes were related to cell division, angiogenesis, and the cell cycle, but the downregulated genes were related to differentiation, such as renal system development (Fig. 2B). We selected the genes whose expression differed by greater than 2-fold in ADPKD RC cell lines compared with that in normal proximal tubule cells and searched for genes with known functions that were highly related to abnormal features and signaling pathways in ADPKD. Among these genes, the chemokine CXCL12, which
A

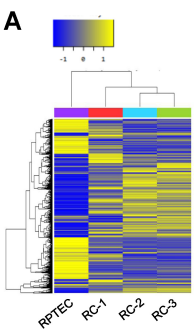

B

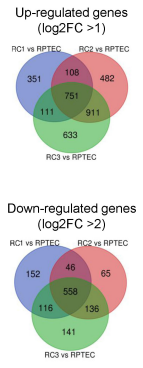

C

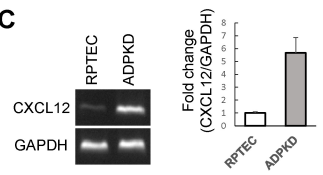

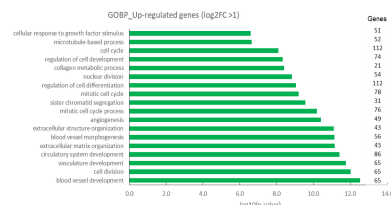

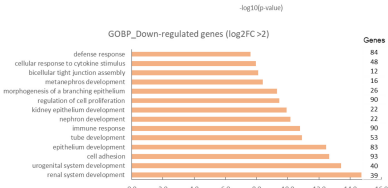

D

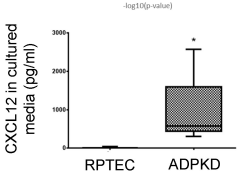

Fig. 2. The chemokine $\mathrm{CXCL12}$ was upregulated and secreted in ADPKD RC cells. (A) Heatmap of genes whose expression varied between the RPTEC/TERT1 normal proximal tubular cell line from the American Type Culture Collection (ATCC) and the ADPKD RC cell lines RC-1, RC-2, and RC-3. (B) Venn diagram and Gene Ontology (GO) analysis of the biological process (BP) terms of genes upregulated and downregulated in three ADPKD RC cell lines. (C) mRNA expression analysis of RPTEC/TERT1 and the ADPKD RC cell line RC- 1 by standard reverse transcriptase polymerase chain reaction (RT-PCR). Relative CXCL12 mRNA expression levels in the RPTEC/TERT1 and ADPKD RC cell lines were normalized to GAPDH mRNA expression. (D) CXCX12 levels in the culture media of RPTEC/TERT1 and ADPKD RC cells were examined by ELISA. The culture media were collected after 72 hours. CXCL12 levels were significantly higher in the ADPKD cyst cell line culture medium than in the RPTEC/TERT1 cell culture medium. ${ }^{*} \mathrm{P}<0.05$.

is related to the mTOR signaling pathway, was upregulated in ADPKD RC cell lines compared with its expression in normal proximal tubule cells. Furthermore, we analyzed the CXCL12 functional interaction network using STRING v11.0 (Fig. S1).

To verify CXCL12 upregulation by transcriptome sequencing analysis, we performed standard reverse transcriptase polymerase chain reaction (RT-PCR) and found that CXCL12 mRNA expression was increased in the ADPKD RC cell lines compared with that in normal proximal tubule cells (Fig. 2C). To examine CXCL12 secretion, the protein levels of CXCL12 in cultured media were measured using a commercially available ELISA kit for CXCL12 and found to be significantly increased in the culture media of the ADPKD RC cell lines, which is consistent with the increased CXCL12 mRNA levels (Fig. 2D). Thus, the expression and secretion of CXCL12 were increased in the ADPKD RC cell lines.

CXCL12 secretion was enhanced in ADPKD RC fluids, and CXCL12/CXCR4 might regulate the mTOR signaling pathway in ADPKD

To confirm secretion of the chemokine CXCL12 from renal cyst-lining cells in ADPKD, we investigated CXCL12 protein levels in the RC fluids of ADPKD patients. CXCL12 secretion 
was enhanced in the RC fluids of ADPKD patients compared with the levels found in normal plasma, as shown by ELISA (Fig. 3A). CXCL12 binds to the receptors CXCR4 and/or CXCR7 and activates different signals on multiple signaling pathways, such as the p38, extracellular signal-related kinase (ERK1/2), mTOR and JNK signaling pathways. To investigate the role of CXCL12 in ADPKD RC fluids, we examined the expression of the CXCL12 receptors CXCR4 and CXCR7. CXCR4 was upregulated and the mTOR signaling pathway, which is downstream of the CXCL12/CXCR4 axis, was activated in kidney tissues from ADPKD patients (Fig. 3B and C).

To confirm whether CXCL12 activated mTOR signal pathway via CXCR4, we treated recombinant CXCL12 and CXCR4 antagonist, AMD3100, and found that CXCL12 induced mTOR signal pathway but CXCR4 antagonist blocked (Fig. 3D). Thus, enhanced CXCL12 in RC fluids binds to CXCR4, and the CXCL12/CXCR4 axis may activate the mTOR signaling pathway, which is related to RC growth in ADPKD.

\section{DISCUSSION}

In autosomal dominant polycystic kidney disease (ADPKD),
A

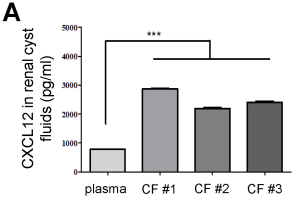

C

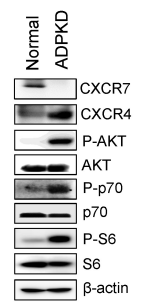

B

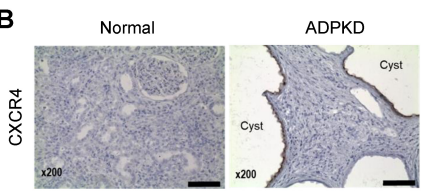

D

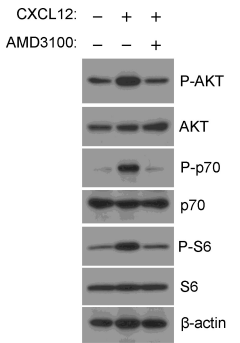

Fig. 3. $\mathrm{CXCL} 12$ levels were enhanced in RC fluids, and the CXCL12/ CXC chemokine receptor 4 (CXCR4) axis activated the mTOR signaling pathway in ADPKD. (A) CXCL12 levels in RC fluids were determined by ELISA. CXCL12 levels in the cyst fluids (CFs) of ADPKD patients were significantly higher than CXCL12 levels in normal plasma. ${ }^{* * * P}<0.001$. (B) As shown by $\mathrm{IHC}$ analysis of normal kidney and ADPKD kidney tissues, strong CXCR4 staining was observed along the cyst-lining epithelial cells in ADPKD tissues. Scale bar: $100 \mu \mathrm{m}$. (C) CXCR4 was upregulated, and the mTOR signaling pathway, which includes AKT/p70/S6, was activated in ADPKD kidney tissues. (D) CXCL12 activated the mTOR signaling pathway through CXCR4 in the ADPKD RC cell lines. After serum starvation for 24 hours, RC-1 cells were treated with $3 \mathrm{ng} / \mathrm{ml}$ recombinant CXCL12 for 4 hours, resulting in activation of the mTOR signaling pathway, which includes AKT/p70/S6, which was blocked by treatment with 50 $\mu \mathrm{M}$ AMD3100, a CXCR4 antagonist. the formation and growth of renal cysts (RCs) is a typical phenotype. RC cells, but not whole kidney tissues, are very useful to study RC formation and growth in ADPKD (22). Previous researchers immortalized cyst cells using the SV40 T antigen, which induces secondary effects on growth regulatory pathways and polyploidy, resulting in genomic instability (12). We immortalized RC cells using hTERT in this study because the telomerase method induces fewer secondary effects and less genomic instability than the SV40 method.

Previous researchers generated a telomerase-immortalized cyst cell line, but this cell line showed only a single detectable PKD1 mutation and no somatic mutations (20). We identified germline and somatic mutations in immortalized RC cell lines by the direct sequencing of gDNA from blood paired with gDNA from cyst cell lines. By direct PKD1 and PKD2 sequencing, we found a novel PKD1 germline missense mutation, c.8347G >C (p. A2783P), in the RC-3 cell line, and the single amino acid substitution in RC-3 was predicted to most likely be damaging by Polyphen-2, Mutation Taster and SIFT. Both germline and somatic missense mutations were found in the PKD1 receptor for egg jelly (REJ) domain in the RC-3 cell line. Mutations in the REJ domain have been proposed to be significant because the REJ domain is required for cleavage at the $\mathrm{G}$ protein-coupled receptor proteolytic site (GPS). According to the PKD mutation database, more than 100 confirmed pathogenic mutations in the REJ domain have been reported, and 8 confirmed pathogenic mutations in the GPS have been identified. Cleavage at the GPS of PC1 has been suggested as essential to normal PC1 function. Missense mutations in the GPS of PKD1 have been shown to be pathogenic, and GPS cleavage mutants that cannot undergo cleavage have been found to cause the loss of normal PC1 functions, including tubulogenesis and JAK-STAT pathway activation.

We first generated three hTERT-immortalized ADPKD RC cell lines with both germline and somatic mutations. These cyst cell lines are identical to RC cells, and transcriptome sequencing of the ADPKD cyst cell lines will be useful for studying the mechanistic details of RCs in ADPKD.

CXC chemokine receptor 4 (CXCR4) contributes to several cellular activities, including survival, proliferation and adhesion, via binding with CXCL12. Several studies have reported that CXCR4 promotes adhesion, chemotaxis, cell proliferation and activation of downstream signal pathways, such as the extracellular signal-regulated kinase (ERK1/2) and/or mTOR pathways. However, studies of the CXCL12/ CXCR4 axis have focused on cancer, but the role of the CXCL12/CXCR4 axis in ADPKD remains largely unclear.

In this study, we showed enhanced CXCL12 expression and secretion in ADPKD RC cells and increased CXCL12 levels in RC fluids. To confirm whether CXCL12 expression is increased in patients with ADPKD, we searched the Gene Expression Omnibus (GEO) datasets of the National Center for Biotechnology Information (NCBI) (https://www.ncbi.nlm.nih. 
gov/gds/) and found that CXCL12 is increased 2.7-fold (P value $<0.05$ ) in PKD1 RCs in ADPKD patients (accession number GSE7869). Thus, CXCL12 expression is increased in patients with ADPKD.

Cyst formation and growth in ADPKD occur by inactivation of the PKD1 or PKD2 gene through germline and somatic mutations. Germline mutation alone is not sufficient for cyst formation in ADPKD. After somatic mutations, renal epithelial cells with a germline mutation in the PKD1 or PKD2 gene become cyst cell and turn the expression of many genes and signaling pathways related to cyst formation and growth on or off. In this study, CXCL12 may have been activated by PKD1 inactivation through somatic mutations. The CXCL12 receptor CXCR4 was also upregulated in ADPKD kidney tissue, and the mTOR signaling pathway, which is downstream of the CXCL12/CXCR4 axis and related to cyst growth, was activated by CXCL12 via CXCR4 in ADPKD renal cells. Taken together, these results suggest that enhanced CXCL12 in RC fluids might activate the mTOR signaling pathway through CXCR4, indicating that the CXCL12/CXCR4 axis might be involved in cystic epithelial cell proliferation and cyst expansion in ADPKD.

\section{MATERIALS AND METHODS}

\section{Immortalization of ADPKD renal cyst (RC) cells by hTERT} Several single cysts 1-3 cm in diameter were dissected, sectioned with scissors, and then incubated with trypsin/EDTA (Gibco) at $37^{\circ} \mathrm{C}$ for 1 hour. Digested cyst cells were cultured in defined keratinocyte-SFM medium (Gibco) to select epithelial cells and exclude contaminating fibroblast cells. After epithelial cell selection for several weeks, RC cells were cultured and adapted in DMEM with $10 \% \mathrm{FBS}$ at $37^{\circ} \mathrm{C}$ in $5 \% \mathrm{CO}_{2}$.

For immortalization, primary cultured cyst cells were transfected with hTERT by Lipofectamine 2000 (Invitrogen). After transfection, cells were selected with $1 \mathrm{mg} / \mathrm{ml} \mathrm{G} 418$ for two passages and then maintained with $0.5 \mathrm{mg} / \mathrm{ml} \mathrm{G} 418$.

\section{Immunofluorescence}

Cells were grown on chamber slides and fixed with $2 \%$ paraformaldehyde for 10 minutes. After fixation, the cells were permeabilized with $0.1 \%$ Triton X-100 in PBS for 10 minutes, followed by blocking with $1 \%$ BSA in PBS for 30 minutes. Thereafter, the cells were incubated with primary antibodies in blocking buffer ( $5 \%$ FBS/ $0.05 \%$ Triton $\mathrm{X}-100$ in PBS) overnight. We used FITC-conjugated anti-cytokeratin antibody (Sigma) as an epithelial marker, FITC-conjugated anti-lotus tetragonolobus lectin (LTL, Vector Laboratories) as a proximal tubule marker, and rhodamine-conjugated anti-dolichos biflorus agglutinin (DBA, Vector Laboratories) as a collecting duct marker.

\section{Direct sequencing of the PKD1 and PKD2 genes}

Genomic DNA (gDNA) was extracted from whole blood and each RC cells using gDNA purification kit (Qiagen) according to the manufacturer's instructions. Direct sequencing of the PKD1 and PKD2 genes was performed by a Sanger sequencing-based method described by Tan et al. (21). Specific long-range polymerase change reaction (PCR) primers were applied for exons 1-33 of PKD1. The long-range PCR templates for exons 1-33 of PKD1 were then amplified by nested PCR, while exons 34-46 of PKD1 and PKD2 were directly amplified from gDNA and sequenced. All PCR products were sequenced in both directions using amplification primers and a Big Dye Terminator v 1.1 Cycle Sequencing Kit (Applied Biosystems). All primer sequences and PCR conditions in this study have been described previously (21).

\section{RNA preparation and transcriptome sequencing analysis} Total RNA was isolated from ADPKD RC cell lines and RPTEC/TERT1 cells (American Type Culture Collection, ATCC), a renal proximal tubular epithelial cell line immortalized with hTERT used as a control, using the TRIzol method according to the manufacturer's protocol (Qiagen). Transcriptome sequencing analysis was performed by MacroGen, Inc. (www.macrogen. com) with the Illumina HiSequation 2000 platform.

\section{CXCL12 mRNA expression and secretion analysis}

To examine CXCL12 mRNA expression, we performed standard reverse transcriptase polymerase chain reaction (RT-PCR) with cDNA synthesized from $1 \mu \mathrm{g}$ total RNA from RPTEC/TERT1 or the RC cells. The CXCL12 mRNA expression levels were normalized to those of GAPDH. The primer pairs used for this study were as follows: CXCL12-F: 5'-TCAGCCTG AGCTACAGATGC-3' and CXCL12-R: 5'-CTTTAGCTTCGGG TCAATGC-3'; GAPDH-F: 5'-TTGCCATCAATGACCCCTTCA-3' and GAPDH-R: 5'-CGCCCCACTTGATTTTGGA-3'.

To analyze CXCL12 secretion, the culture media from RPTEC/TERT1 cells and RC cell lines were collected after 72 hours of culture, and each supernatant was centrifuged to remove cellular debris. RC fluids were collected from each cyst from ADPKD patients. CXCL12 concentrations in the culture media and cyst fluids (CFs) were measured using a CXCL12 ELISA kit (R\&D system) according to the manufacturer's protocol.

\footnotetext{
Western blot (WB) analysis of kidney tissue

Normal kidney tissues and ADPKD patient kidney tissues were homogenized and then lysed with RIPA buffer. Protein lysates of the kidney tissues were separated by $12 \%$ SDS-PAGE and transferred to PVDF membranes. The membranes were incubated in blocking buffer $(3 \%$ nonfat dry milk in Tris-buffered saline and $0.1 \%$ Tween 20 (TBST)) for 1 hour and then probed with primary antibodies overnight at $4^{\circ} \mathrm{C}$. We used the primary antibodies anti-CXC chemokine receptor 4 (CXCR4) (Abcam), anti-CXC chemokine receptor 7 (CXCR7) (Abcam), anti-phospho-AKT (Cell Signaling Technology),
} 
anti-AKT (Cell Signaling Technology), anti-phospho-p70 (Cell Signaling Technology), anti-p70 (Cell Signaling Technology), anti-phospho-S6 (Cell Signaling Technology), anti-S6 (Cell Signaling Technology), and anti- $\beta$-actin (Sigma) for WB analysis. After washing three times with TBST, the blots were incubated in peroxidase-labeled secondary antibody for 1 hour. Then, the blots were washed three times with TBST, and the probed proteins were detected using an enhanced chemiluminescence $(\mathrm{ECL})$ detection system (GE Healthcare).

\section{CXCR4 immunohistochemistry (IHC)}

CXCR4 expression in ADPKD kidney tissues was determined by IHC. Kidney tissues were fixed in $4 \%$ paraformaldehyde and embedded in paraffin. Paraffinized sections were deparaffinized with xylene, followed by rehydration using an ethanol gradient. Antigen retrieval was conducted in citrate buffer $\left(\mathrm{pH} \mathrm{6.0)}\right.$ at $95-100^{\circ} \mathrm{C}$ for 20 minutes. After blocking with $2 \%$ normal goat serum, anti-CXCR4 (Abcam) was added to the slide. The sections were then incubated with an HRPlabeled secondary antibody (Zytomed System) and visualized with 3,3'-diaminobenzidine (DAB, Vector Laboratories). The slides were counterstained with hematoxylin (Vector Laboratories) and analyzed under an Olympus microscope BX51.

\section{ACKNOWLEDGEMENTS}

This research was supported by the National Research Foundation (NRF) funded by the Korean government (MSIT) (NRF-2016R1A2B4015516 and NRF-2016M3A9B6903411).

\section{CONFLICTS OF INTEREST}

The authors have no conflicting interests.

\section{REFERENCES}

1. Peters DJ and Breuning MH (2001) Autosomal dominant polycystic kidney disease: modification of disease progression. Lancet 358, 1439-1444

2. Harris PC and Torres VE (2009) Polycystic kidney disease. Annu Rev Med 60, 321-337

3. Ghata J and Cowley BD Jr (2017) Polycystic Kidney Disease. Compr Physiol 7, 945-975

4. Sutters M and Germino GG (2003) Autosomal dominant polycystic kidney disease: molecular genetics and pathophysiology. J Lab Clin Med 141, 91-101

5. Watnick TJ, Piontek KB, Cordal TM et al (1997) An unusual pattern of mutation in the duplicated portion of PKD1 is revealed by use of a novel strategy for mutation detection. Hum Mol Genet 6, 1473-1481

6. Rossetti S, Hopp K, Sikkink RA et al (2012) Identification of gene mutations in autosomal dominant polycystic kidney disease through targeted resequencing. J Am Soc Nephrol 23, 915-933

7. Pei Y (2001) A "two-hit" model of cystogenesis in autosomal dominant polycystic kidney disease? Trends Mol Med 7, 151-156

8. Bodnar AG, Ouellette M, Frolkis M et al (1998) Extension of life-span by introduction of telomerase into normal human cells. Science 279, 349-352

9. Counter CM, Hahn WC, Wei W et al (1998) Dissociation among in vitro telomerase activity, telomere maintenance, and cellular immortalization. Proc Natl Acad Sci U S A 95, 14723-14728

10. Lee KM, Choi KH and Ouellette MM (2004) Use of exogenous hTERT to immortalize primary human cells. Cytotechnology 45, 33-38

11. Ouellette MM, McDaniel LD, Wright WE, Shay JW and Schultz RA (2000) The establishment of telomeraseimmortalized cell lines representing human chromosome instability syndromes. Hum Mol Genet 9, 403-411

12. Hein J, Boichuk S, Wu J et al (2009) Simian virus 40 large $\mathrm{T}$ antigen disrupts genome integrity and activates a DNA damage response via Bub1 binding. J Virol 83, 117-127

13. Shirozu M, Nakano T, Inazawa J et al (1995) Structure and chromosomal localization of the human stromal cellderived factor 1 (SDF1) gene. Genomics 28, 495-500

14. Nagasawa T (2014) CXC chemokine ligand 12 (CXCL12) and its receptor CXCR4. J Mol Med 92, 433-439

15. Sánchez-Martín L, Sánchez-Mateos P, Cabañas C (2013) CXCR7 impact on CXCL12 biology and disease. Trends Mol Med 19, 12-22

16. Liekens S, Schols D and Hatse S (2010) CXCL12-CXCR4 axis in angiogenesis, metastasis and stem cell mobilization. Curr Pharm Des 16, 3903-3920

17. Guyon A (2014) CXCL12 chemokine and its receptors as major players in the interactions between immune and nervous systems. Front Cell Neurosci 8, 65

18. Ghanem I, Riveiro ME, Paradis V, Faivre S, de Parga PM and Raymond E (2014) Insights on the CXCL12-CXCR4 axis in hepatocellular carcinoma carcinogenesis. Am J Transl Res 6, 340-352

19. Scala S (2015) Molecular Pathways: Targeting the CXCR4-CXCL12 Axis--Untapped Potential in the Tumor Microenvironment. Clin Cancer Res 21, 4278-4285

20. Herbert BS, Grimes BR, Xu WM et al (2013) A telomerase immortalized human proximal tubule cell line with a truncation mutation (Q4004X) in polycystin-1. PLoS One 8, e55191

21. Tan YC, Blumenfeld JD, Anghel R et al (2009) Novel method for genomic analysis of PKD1 and PKD2 mutations in autosomal dominant polycystic kidney disease. Hum Mutat 30, 264-273

22. Jo HS, Eum WS, Park EY et al (2017) Effects of PEP-1-FK506BP on cyst formation in polycystic kidney disease. BMB Rep 50, 460-465 